\title{
Odor naming and interpretation performance in 881 schizophrenia subjects: association with clinical parameters
}

\author{
Anne Kästner ${ }^{1 \dagger}$, Dörthe Malzahnn ${ }^{2 \dagger}$, Martin Begemann ${ }^{1}$, Constanze Hilmes ${ }^{1}$, Heike Bickeböller ${ }^{2}$
} and Hannelore Ehrenreich ${ }^{1,3^{*}}$

\begin{abstract}
Background: Olfactory function tests are sensitive tools for assessing sensory-cognitive processing in schizophrenia. However, associations of central olfactory measures with clinical outcome parameters have not been simultaneously studied in large samples of schizophrenia patients.

Methods: In the framework of the comprehensive phenotyping of the GRAS (Göttingen Research Association for Schizophrenia) cohort, we modified and extended existing odor naming (active memory retrieval) and interpretation (attribute assignment) tasks to evaluate them in 881 schizophrenia patients and 102 healthy controls matched for age, gender and smoking behavior. Associations with emotional processing, neuropsychological test performance and disease outcome were studied.

Results: Schizophrenia patients underperformed controls in both olfactory tasks. Odor naming deficits were primarily associated with compromised cognition, interpretation deficits with positive symptom severity and general alertness. Contrasting schizophrenia extreme performers of odor interpretation (best versus worst percentile; $\mathrm{N}=88$ each) and healthy individuals $(\mathrm{N}=102)$ underscores the obvious relationship between impaired odor interpretation and psychopathology, cognitive dysfunctioning, and emotional processing (all $p<0.004$ ).

Conclusions: The strong association of performance in higher olfactory measures, odor naming and interpretation, with lead symptoms of schizophrenia and determinants of disease severity highlights their clinical and scientific significance. Based on the results obtained here in an exploratory fashion in a large patient sample, the development of an easy-to-use clinical test with improved psychometric properties may be encouraged.
\end{abstract}

Keywords: Odor naming, Higher olfactory processing, Odor interpretation, Positive symptoms, Cognition

\section{Background}

The sense of smell and its relation to neurological and psychiatric diseases is a field of growing interest in clinical research. Like other neuropsychological measures, it provides the opportunity to assess brain function in a non-invasive way [1]. Central neural circuits underlying olfactory function can be mapped to temporolimbic and frontal brain regions [2]. Dysfunctional connectivity

\footnotetext{
* Correspondence: ehrenreich@em.mpg.de

${ }^{\dagger}$ Equal contributors

${ }^{1}$ Clinical Neuroscience, Max Planck Institute of Experimental Medicine, Hermann-Rein-Str.3, 37075 Göttingen, GERMANY

${ }^{3}$ DFG Research Center for Nanoscale Microscopy \& Molecular Physiology of the Brain (CNMPB), Göttingen, Germany

Full list of author information is available at the end of the article
}

in these olfactory structures has been consistently implicated in the affective and cognitive symptomatology of schizophrenia patients [3]. Consequently, olfactory tasks serve as behavioral probes to assess the structural and functional integrity of neural substrates underlying disturbed sensory-cognitive and emotional processing in schizophrenia [3].

Olfactory input triggers cognitive events, often in form of autobiographical memories [4-6]. Tests measuring olfactory cognition address the disturbed integration of olfactory input into cognitive processing. They share the common principle of administering an odorant prior to the execution of related mental operations with varying degrees of difficulty [7]. The more demanding the olfactory 
task, the more likely the contribution of higher-order cortical olfactory circuits. Where in the olfactory projection cascade difficulties for schizophrenia individuals arise is still unclear [8]. Nevertheless, evidence of a peripheral deficit in terms of abnormal olfactory receptor function accumulated over the past years $[9,10]$. Profound problems consistently emerge in schizophrenia patients when the olfactory task requires basal cognitive operations like the passive recognition of a presented odor from lists of distractors (passive odor identification) [11-13], or when a delay between odor presentation and identification is introduced (odor memory) [4,14].

The identification and explicit naming of odorants (active odor naming) is a complex task requiring correct smell encoding, semantic memory, and selection of the most appropriate denomination [15]. Saoud and coworkers delivered first evidence of an odor naming deficit in a small sample $(\mathrm{N}=24)$ of male schizophrenia patients [16]. An implication of dorsolateral prefrontal executive functioning networks in this task was supported by its correlation with Wisconsin Card Sorting Test performance [16]. Surprisingly, clinical variables like PANSS scores or age at onset did not correlate with odor naming ability in this study [16]. Although less cognitively demanding, associations with schizophrenia psychopathology including cognition have been consistently shown for passive odor identification $[8,17-19]$. The missing link between PANSS scores and odor naming ability in the study by Saoud and coworkers likely reflects a lack of statistical power due to the very small sample size $(\mathrm{N}=24)$. As it is a very elegant and naturalistic measure of the integrity of prefrontal networks, we decided to extend in the present study the work reported by Saoud and colleagues by evaluating odor naming performance and its relationship with clinical and cognitive variables in a large sample of schizophrenia patients.

Olfactory stimuli do not only induce cognitive activity, they immediately evoke emotional reactions [20]. To explore the nature of affective states in response to different scents, available olfactory function tests require the judgement of various qualities of given odors. Schizophrenia subjects are less accurate when deciding whether an odor is edible, pleasant or familiar as compared to healthy controls [21-24]. It is known that the rating of gustatory or semantic properties of odors facilitates their subsequent naming in healthy control subjects [25]. No study has ever investigated whether schizophrenia patients have difficulties in judging gustatory (sweet, hot) or semantic properties (association of an odor with its context, i.e. technical or natural) of odors beyond familiarity, hedonicity and edibility ratings (odor interpretation). Moreover, it has not been evaluated how the inability to accurately interpret olfactory stimuli relates to schizophrenia symptomatology.
As a part of the very comprehensive phenotyping of the GRAS cohort [26], odor interpretation and naming was evaluated in 881 schizophrenia patients and 102 healthy matched controls. In the present study we assessed odor naming and interpretation ability using diverse odors typical for the 'olfactory environment' of German subjects as well as descriptive attributes (hedonic, gustatory and semantic). We evaluated the relationship of olfactory task performance with relevant symptom domains and clinical outcome measures in the whole schizophrenia sample $(\mathrm{N}=881)$ and, subsequently, in extreme groups of odor interpretation performance (best versus worst percentile), and compared them to healthy individuals $(\mathrm{N}=102)$.

\section{Method}

\section{Subjects}

\section{Schizophrenia subjects}

The present study (GRAS project) was approved by ethics committees of the Georg-August-University Göttingen (master committee) and collaborating centers. Detailed phenotyping of the GRAS sample [26] contained odor naming and odor interpretation tasks (Figure 1A), administered to 999 schizophrenia patients after written informed consent. Present analyses excluded all non-native German speakers $(\mathrm{N}=89)$, all patients with known anosmia $(\mathrm{N}=14$, neurological conditions e.g. head injury, or cold) and 6 patients with missing data. Nine patients were excluded as non-admissible based on performance in entry and odor naming tasks (Figure 1B). Data analyses were based on the remaining 881 patients.

\section{Healthy controls}

As comparison group, 103 healthy subjects matched for age, gender and smoking status (smoker: yes/no) were recruited by public announcements and gave written informed consent. They were free of any physical, neurological and psychiatric disorder, and had no relatives with a history of neuropsychiatric diseases. One individual was excluded due to his performance in entry and odor naming tasks. Data analyses were based on 102 controls.

\section{Measures}

\section{Olfaction}

Odorants The University of Pennsylvania Smell Identification Test [27] (UPSIT) is the olfactory function test most widely applied clinically and scientifically. It has been used as validation criterion for newly developed olfactory tests [28]. A total of 10 different scents (scratch and sniff format) were selected from this battery (for detailed description of the administration procedure see [11]). Odorants were chosen by an experienced psychiatrist based on following criteria: (I) For the entry odor task, odors had to be easily identifiable (low item difficulty) to minimize contribution of higher cognition to task performance. For the naming 


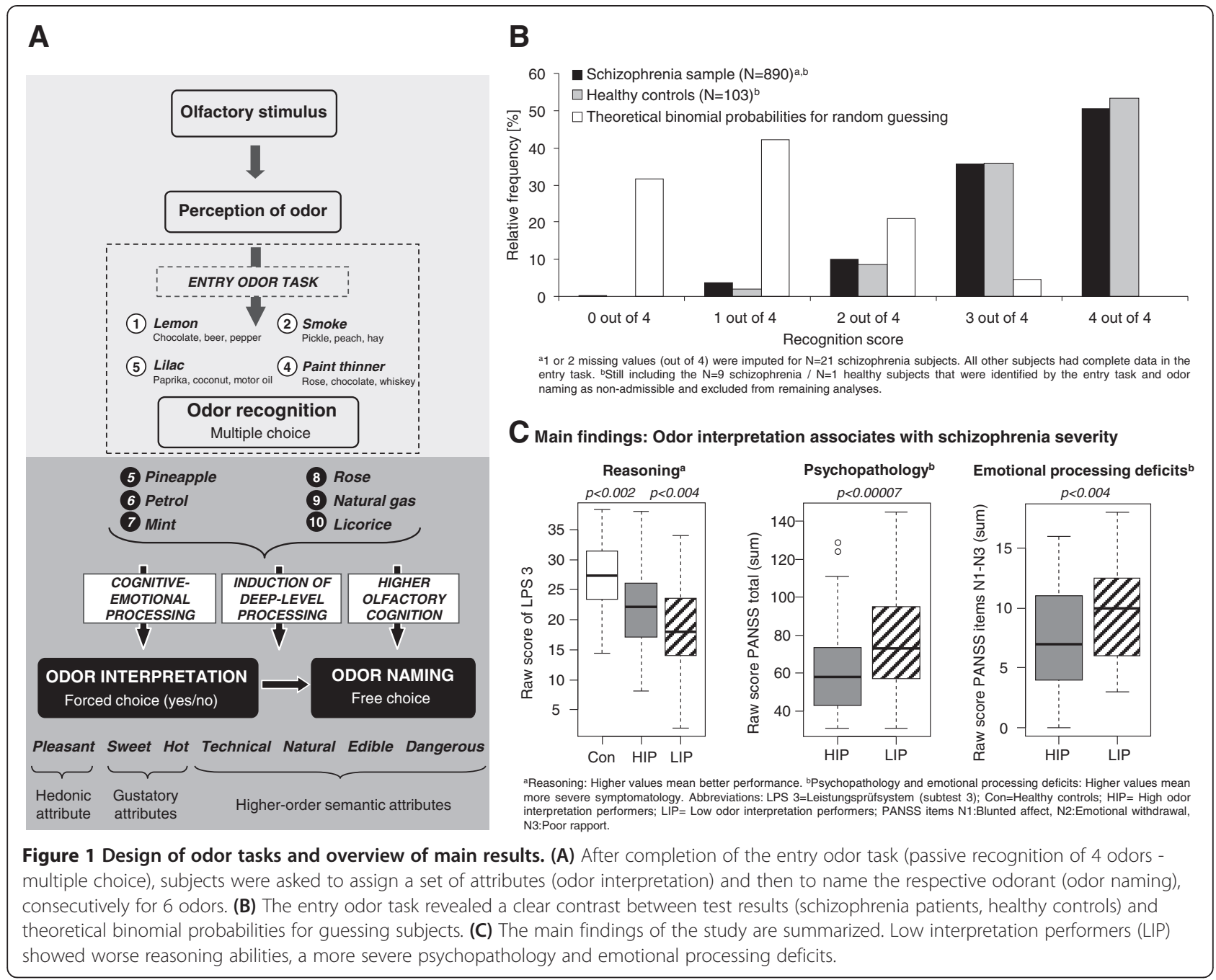

task, odorants were selected to cover a broad range of difficulties to differentiate between subjects along the whole spectrum of cognitive abilities. For odor interpretation, items of low difficulty were chosen due to the expected profound interpretation deficit in schizophrenia. (II) The odors had to be of high ecological validity to German subjects. (III) To balance avoidance of redundancy (due to time limitations) and reliability, odors were selected to represent diverse associated contexts (e.g. rose and petrol) and attribute overlap (both pineapple and licorice are pleasant and sweet). Although this study was not designed to introduce a new ready-to-be-used olfaction test, preliminary item characteristics and psychometric properties are provided in Table 1.

Entry odor task To be able to interpret odor naming and interpretation results as central processing deficits, an entry odor task (Figure 1A; maximum score 4) was introduced. A total of 4 consecutively presented odors are chosen from 4 alternative descriptors each (multiple choice, brief passive odor identification [11]) by pointing to or naming the correct term on the scoring sheet. The entry task should identify potentially anosmic subjects unaware of their condition or failing to report it prior to performing odor naming and interpretation tasks. The 4 odorants lemon, smoke, lilac and paint thinner were chosen to be easily identifiable (Table 1) and representative of all attributes contained in the odor interpretation task. Lemon was selected to cover the attributes sweet and edible, lilac is an odor judged as pleasant and natural, smoke is hot and dangerous and paint thinner is often associated with a technical context. Figure 1B shows that theoretical binomial probabilities for random guessing (in case of anosmia or pronounced cognitive impairment, assuming equal multiple-choice success probabilities of 0.25 for all 4 odors) markedly differed from relative recognition score frequencies. The vast majority of patients and controls recognized 3-4 odors correctly whereas $95 \%$ of guessing subjects would recognize $<3$ odors correctly in the entry task. Simultaneously, guessing would result in an odor 
Table 1 Psychometric properties of odor interpretation and odor naming tasks in the schizophrenia sample ( $\mathrm{N}=881$ )

\begin{tabular}{lcc}
\hline & Odor naming $^{\mathbf{a}}$ & Odor inter \\
\hline Item difficulty & (weighted $\%$ correct naming $\pm \mathbf{9 5 \% C l )}$ \\
Pineapple & $21.71 \pm 0.01$ & $73.0 \pm 1.7$ \\
Petrol & $38.67 \pm 0.02$ & $78.2 \pm 1.3$ \\
Mint & $76.35 \pm 0.03$ & $78.0 \pm 1.2$ \\
Rose & $39.99 \pm 0.02$ & $80.2 \pm 1.3$ \\
Natural gas & $16.61 \pm 0.02$ & $70.9 \pm 1.6$ \\
Licorice & $54.48 \pm 0.03$ & $82.3 \pm 1.3$
\end{tabular}

Item discrimination, part-whole corrected [95\%Cl]

$\begin{array}{lll}\text { Pineapple } & 0.24[0.18,0.30] & 0.21[0.15,0.27] \\ \text { Petrol } & 0.25[0.19,0.31] & 0.25[0.19,0.31] \\ \text { Mint } & 0.30[0.24,0.36] & 0.27[0.20,0.33] \\ \text { Rose } & 0.28[0.21,0.34] & 0.18[0.11,0.24] \\ \text { Natural gas } & 0.13[0.07,0.20] & 0.16[0.09,0.22] \\ \text { Licorice } & 0.27[0.20,0.33] & 0.20[0.14,0.27]\end{array}$

$\begin{array}{ll}\text { Pleasant } & 81.7 \pm 1.2 \\ \text { Sweet } & 72.6 \pm 1.1 \\ \text { Hot } & 69.5 \pm 1.4 \\ \text { Technical } & 81.3 \pm 1.1 \\ \text { Natural } & 75.1 \pm 1.3 \\ \text { Edible } & 80.4 \pm 1.2 \\ \text { Dangerous } & 79.5 \pm 1.1\end{array}$

Item discrimination, not part-whole corrected $[95 \% \mathrm{Cl}]$

$\begin{array}{lll}\text { Pineapple } & 0.41[0.35,0.46] & 0.57[0.52,0.61] \\ \text { Petrol } & 0.50[0.45,0.54] & 0.53[0.48,0.58] \\ \text { Mint } & 0.60[0.55,0.64] & 0.52[0.47,0.57] \\ \text { Rose } & 0.56[0.51,0.61] & 0.45[0.40,0.50] \\ \text { Natural gas } & 0.33[0.26,0.39] & 0.50[0.45,0.55] \\ \text { Licorice } & 0.65[0.62,0.69] & 0.48[0.43,0.53]\end{array}$

$\begin{array}{ll}\text { Pleasant } & 0.46[0.40,0.51] \\ \text { Sweet } & 0.33[0.27,0.39] \\ \text { Hot } & 0.18[0.11,0.24] \\ \text { Technical } & 0.58[0.53,0.62] \\ \text { Natural } & 0.45[0.40,0.50] \\ \text { Edible } & 0.52[0.47,0.56] \\ \text { Dangerous } & 0.52[0.47,0.56]\end{array}$

Pleasant $\quad 0.63[0.59,0.67]$

Sweet $\quad 0.52[0.47,0.56]$

Hot $\quad 0.44[0.38,0.49]$

Technical $\quad 0.72[0.69,0.75]$

Natural $\quad 0.64[0.60,0.68]$

Edible $\quad 0.68[0.64,0.71]$

Dangerous $\quad 0.66[0.62,0.70]$

Internal consistency (standardized Cronbach's a, equals average split-half reliability)

naming score of 0 (free choice test) which is extremely unlikely (Figure 2B). Consequently, subjects were excluded from analysis when they had less than 3 out of 4 correctly recognized odors in the entry task and an odor naming score of 0 (9 schizophrenia subjects, 1 healthy control subject) because of potentially experiencing difficulties at the peripheral processing level. Clearly, this approach risks excluding cognitively extremely impaired individuals. Indeed, excluded individuals cognitively underperformed the 881 schizophrenia subjects ( 881 subjects: $-0.03 \pm 0.78$; 9 excluded subjects: $-0.92 \pm 0.54$; mean \pm SD of higher cognition composite; see below).

Odor naming and odor interpretation The odor naming task (maximum score 18) measures the correct naming of the 6 odors pineapple, petrol, mint, rose, natural gas and licorice (free choice; Figure 1A). Three points were scored for the correct name, 1 point if the subject provided an item belonging to the same semantic category (e.g. pineapple: 3 points for 'pineapple', 1 point for 'fruit'). Before naming an odor, probands had to decide whether it matched the 7 attributes pleasant (hedonic judgement), sweet \& hot (gustatory judgement), technical, natural, edible and dangerous (semantic judgement) (forced choice: 'yes' or 'no' answer; odor interpretation; same set of odors as for naming) (Figure 1A). One point was scored for each attribute assignment consistent with the predefined profile (maximum score $42=6 \times 7$ ). Although task reliability improves with number of items [29], we had to restrict it to 6 items for time-economic reasons (olfactory tasks being only a fraction of the GRAS examination procedure [26]).

\section{Disease-relevant possible confounders}

As unspecific but disease-relevant (overall severity) possible confounders, duration of disease, number of hospitalizations and medication status (chlorpromazine equivalents) were selected. 


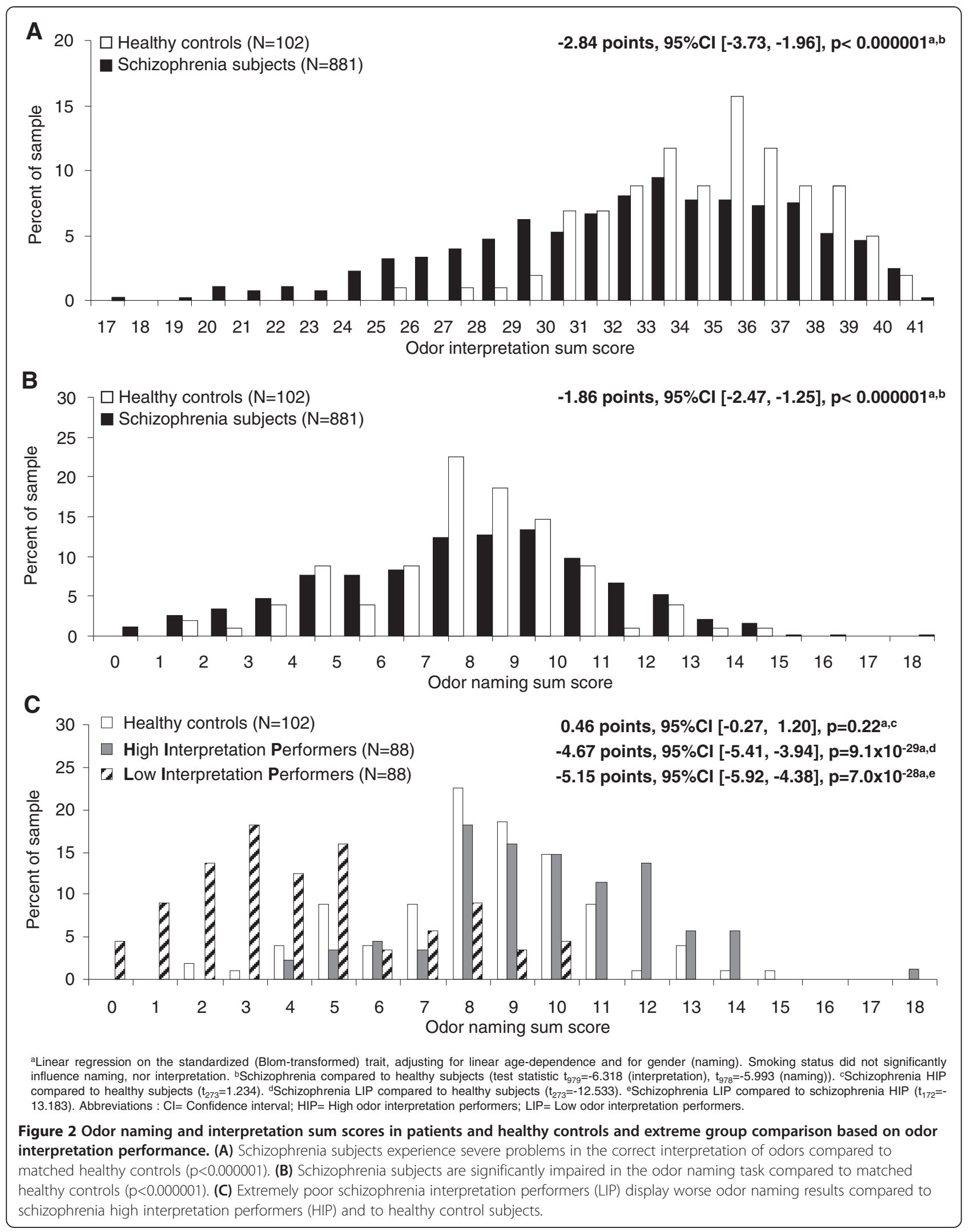




\section{Functional outcome, disease severity, psychopathology, cognition and emotional processing}

To address schizophrenia relevant target domains, age-atprodrome, general assessment of functioning (GAF) scale, PANSS (positive and negative syndrome scale) subscales [30], a higher cognition composite (comprising neuropsychological measures listed in Table 2), and the alertness subtest (mean of tonic and phasic alertness) of TAP (Testbatterie für Aufmerksamkeitsprüfung [31], English translation: Test battery for attention) were included. MacQuarrie dotting and tapping tests [32] were integrated into a fine motor function composite. The MehrfachwahlWortschatz-Test [33] (MWTB, English translation: Multiple Choice Vocabulary Test of Intelligence) covered premorbid intelligence. Emotional processing was operationalized as sum score of 'blunted affect,' 'emotional withdrawal' and 'poor rapport' of PANSS, as previous studies have shown that amygdala responses to facial emotional expression correlate with the negative subscale of PANSS [34]. Higher scores represent worse outcome in PANSS but better performance in GAF and neuropsychological tests. Healthy controls completed odor naming and

Table 2 Presentation of high (HIP) and low (LIP) interpretation performer profiles with respect to higher cognition composite components and emotional processing items

\begin{tabular}{|c|c|c|c|c|c|c|}
\hline & & \multicolumn{3}{|c|}{ Descriptive } & \multicolumn{2}{|c|}{ LIP compared to HIP } \\
\hline & & SZ & LIP & HIP & Effect & $p$ \\
\hline & & & & & {$[95 \% \mathrm{Cl}]$} & (Statistic) \\
\hline \multicolumn{7}{|c|}{ Higher cognition composite components } \\
\hline \multirow[t]{2}{*}{ Reasoning ${ }^{c}$} & mean $\pm S D$ & $20.8 \pm 6.6$ & $18.2 \pm 6.7$ & $21.5 \pm 6.5$ & -2.61 & $3.6 \times 10^{-3}$ \\
\hline & range & $(2-38)$ & $(2-34)$ & $(8-38)$ & {$[-4.35,-0.86]$} & $\left(t_{164}=-2.951^{\mathrm{a}}\right)$ \\
\hline \multirow[t]{3}{*}{ Executive functioning $^{c}$} & median & -56.5 & -79.0 & -47.0 & -24.77 & 0.082 \\
\hline & interquartile & {$[-103.0,-36.0]$} & {$[-139.0,-47.0]$} & {$[-122.0,-34.0]$} & {$[-52.69,3.15]$} & $\left(\mathrm{t}_{155}=-1.753^{\mathrm{a}}\right)$ \\
\hline & range & $(-868-40)$ & $(-680--9)$ & $(-563--10)$ & & \\
\hline \multirow[t]{2}{*}{ Working memory ${ }^{c}$} & mean $\pm S D$ & $13.1 \pm 3.9$ & $11.7 \pm 4.4$ & $13.6 \pm 3.9$ & -1.25 & 0.049 \\
\hline & range & $(1-24)$ & $(4-22)$ & $(4-24)$ & {$[-2.50,-0.00]$} & $\left(\mathrm{t}_{158}=-1.981^{\mathrm{a}}\right)$ \\
\hline \multirow[t]{2}{*}{ Processing speed ${ }^{c}$} & mean $\pm \mathrm{SD}$ & $38.4 \pm 13.2$ & $32.5 \pm 13.7$ & $39.3 \pm 13.5$ & -4.52 & 0.011 \\
\hline & range & $(4-88)$ & $(5-68)$ & $(6-71)$ & {$[-7.99,-1.04]$} & $\left(\mathrm{t}_{167}=-2.566^{\mathrm{a}}\right)$ \\
\hline \multirow[t]{2}{*}{$V$ erbal memory ${ }^{c}$} & mean $\pm S D$ & $42.0 \pm 12.6$ & $37.3 \pm 12.5$ & $43.0 \pm 14.0$ & -3.38 & 0.083 \\
\hline & range & $(6-72)$ & $(11-62)$ & $(6-70)$ & {$[-7.22,0.45]$} & $\left(\mathrm{t}_{161}=-1.742^{\mathrm{a}}\right)$ \\
\hline \multirow[t]{3}{*}{ Divided attention ${ }^{c}$} & median & -725 & -764 & -714 & 4.14 & 0.84 \\
\hline & interquartile & {$[-806,-659]$} & {$[-833,-651]$} & {$[-797,-647]$} & {$[-35.50,43.78]$} & $\left(t_{158}=0.206^{a}\right)$ \\
\hline & range & $(-1663--355)$ & $(-1167--448)$ & $(-1160--416)$ & & \\
\hline \multicolumn{7}{|c|}{ Emotional processing items } \\
\hline Blunted affect & median & 3 & 4 & 3 & & $7.5 \times 10^{-3}$ \\
\hline \multirow[t]{2}{*}{ (PANSS N1) } & interquartile & {$[2,4]$} & {$[3,5]$} & {$[2,4]$} & - & $\left(W=2947^{b}\right)$ \\
\hline & range & $(1-7)$ & $(1-7)$ & $(1-6)$ & & \\
\hline Emotional withdrawal & median & 3 & 3 & 3 & & 0.029 \\
\hline \multirow[t]{2}{*}{ (PANSS N2) } & interquartile & {$[1,4]$} & {$[1,4]$} & {$[1,4]$} & - & $\left(W=3119^{b}\right)$ \\
\hline & range & $(1-7)$ & $(1-7)$ & $(1-6)$ & & \\
\hline Poor rapport & median & 2 & 3 & 2 & & $3.1 \times 10^{-3}$ \\
\hline \multirow[t]{2}{*}{ (PANSS N3) } & interquartile & {$[1,3]$} & {$[1,4]$} & {$[1,3]$} & - & $\left(W=2875^{b}\right)$ \\
\hline & range & $(1-7)$ & $(1-6)$ & $(1-5)$ & & \\
\hline
\end{tabular}

Multiple testing adjusted significances are set in boldface ( $\mathrm{p} \leq 0.0083$ for components of higher cognition composite, $p \leq 0.0167$ for emotional processing items). All other $\mathrm{p}$-values $\leq 0.05$ in italic. ${ }^{\mathrm{a}} \mathrm{t}$-Statistic $\mathrm{t}_{\mathrm{df}}$ with $\mathrm{df}$ degrees of freedom for estimation of difference in means (employing a linear model on Blom-transformed trait). ${ }^{b}$ Wilcoxon rank sum test. 'Adjusted for age and PANSS negative. Reasoning ability was measured by LPS3, executive functioning is represented as difference of execution times between TMTA and TMTB, working memory was assessed by BZT, processing speed by ZST, verbal memory by VLMT and divided attention by negative execution time $(-\mathrm{d} 3 \mathrm{mdg})$ of TAP. In this table, and when building the cognition composite, all measures were presented such that larger values represent better cognitive performance. Abbreviations: $\mathrm{SZ}=$ Descriptive statistics for schizophrenia sample $(\mathrm{N}=881)$; $\mathrm{HIP}=\mathrm{High}$ interpretation performers ( $\mathrm{N}=88$ ); $\mathrm{LIP}=$ Low interpretation performers $(\mathrm{N}=88)$; $\mathrm{LPS} 3=$ Subtest 3 of the Leistungsprüfsystem, a German test covering Thurstone's primary mental abilities; TMTA/B= Trail Making Test A and B; BZT=Letter Number Sequencing; ZST= Digit Symbol Coding; VLMT= Verbal Learning and Memory Test; TAP=Testbatterie für Aufmerksamkeitsprüfung, English translation: Test battery for attention. All neuropsychological measures are cited in Ribbe et al. 2010. 
interpretation tasks and 4 neuropsychological tests (LPS 3 [35]; MWTB [33]; subtests dotting and tapping subtests of the MacQuarrie test for mechanical ability [32]). All tests are cited in [26].

\section{Statistical analyses}

To test for associations of odor naming and interpretation performance with disease-relevant symptom domains, multiple linear regression was applied within the schizophrenia sample (Table 3). The model simultaneously tested for linear dependence of the target (odor naming or odor interpretation performance) on a set of multiple predictor variables (cognitive performance, symptom severity and control variables) while adjusting for gender (odor naming) and for linear age-dependence (odor naming and odor interpretation). Displayed regression coefficients are mutually adjusted for the respective other predictors. All variables were a priori standardized such that regression coefficients quantify relative association strengths interpretable in analogy to Cohen's d, displaying mutually adjusted relative association strengths interpretable in analogy to Cohen's d). Sum scores for higher cognition and fine motor performance are the mean of standardized neuropsychological measures in these domains (larger values represent better performance). Rank-based Blom transformation [36] was applied to standardize all measures by transforming them into standard normally distributed surrogates prior to sum score computation. This maintained the order of the data, but removed skewness from variable distributions. Group comparisons between healthy and schizophrenia subjects (Figure 2A-B), and schizophrenia subgroups (Figure 1C, Figure 2C and Tables 2, 4 and 5) were tested by linear regression (for standardized normally distributed quantitative targets, adjusting for covariates where indicated), Fisher's exact test (for binary targets gender, smoking and genetic risk status), or non-parametric Wilcoxon rank sum test (for quantitative targets which could not be standardized to normal distribution). For linear model analyses, the target variable is denoted in the table row, respectively. The tested predictor was class membership, adjusting for linear dependence on age, PANSS negative and for gender where indicated. Standardized regression coefficients (with 95\% confidence limits) were converted to the original variable scale for ease of interpretation by multiplication with the raw data standard deviation of the target variable within the respective data. Multiple-testing was accounted for by Bonferroni correction and closed testing principle (Table 4). All p-values are two-sided.

\section{Results}

Influence of age, smoking and gender on odor naming and interpretation

Increasing age is associated with a decline in odor naming (average -0.05 points/year, $\mathrm{p}=2.2 \times 10^{-11}$, test statistic $t_{978}=-6.770$ ) and interpretation performance (average -0.06 points/year, $\mathrm{p}=1.6 \times 10^{-8}, \mathrm{t}_{979}=-5.697$ ) likewise in schizophrenia and healthy individuals (no significant difference). Smoking status had no influence on both central olfactory measures. Women proved to be slightly superior to men

Table 3 Odor naming is associated with cognition and odor interpretation with severity of positive symptoms and alertness in schizophrenia subjects $(\mathrm{N}=\mathbf{8 8 1})$

\begin{tabular}{|c|c|c|}
\hline & Odor naming $^{a}$ & Odor interpretation $^{\mathrm{a}}$ \\
\hline \multirow[t]{2}{*}{ Higher cognition composite ${ }^{b}$} & 0.165 & 0.057 \\
\hline & $\left(t_{813}=2.347, p=0.019\right)$ & $\left(t_{814}=0.789, p=0.430\right)$ \\
\hline \multirow[t]{2}{*}{ Alertness (TAP) } & 0.144 & 0.196 \\
\hline & $\left(t_{813}=3.401, p=0.001\right)$ & $\left(t_{814}=4.635, p=0.000\right)$ \\
\hline \multirow[t]{2}{*}{ PANSS negative } & -0.112 & -0.045 \\
\hline & $\left(t_{813}=-2.717, p=0.007\right)$ & $\left(\mathrm{t}_{814}=-1.073, \mathrm{p}=0.283\right)$ \\
\hline \multirow[t]{2}{*}{ PANSS positive } & -0.055 & -0.107 \\
\hline & $\left(\mathrm{t}_{813}=-1.407, \mathrm{p}=0.160\right)$ & $\left(t_{814}=-2.701, p=0.007\right)$ \\
\hline \multirow[t]{2}{*}{ Fine motor function composite ${ }^{c, d}$} & -0.010 & 0.017 \\
\hline & $\left(t_{813}=-0.210, p=0.834\right)$ & $\left(t_{814}=0.336, p=0.737\right)$ \\
\hline \multirow[t]{2}{*}{ Premorbid intelligence (MWTB) ${ }^{d}$} & 0.071 & 0.059 \\
\hline & $\left(t_{813}=1.761, p=0.079\right)$ & $\left(t_{814}=1.420, p=0.156\right)$ \\
\hline
\end{tabular}

${ }^{a}$ Relative association strength of domains with odor naming and odor interpretation ( $t$-statistic $t_{d f}$ with df degrees of freedom, $p$ value). Multiple linear regression model containing linear terms for all six predictor variables (table rows) and adjusting for age (odor naming and odor interpretation) and gender (odor naming), significances (Bonferroni $\mathrm{p} \leq 0.025$ ) in boldface. ${ }^{\mathrm{b}}$ The higher cognition composite includes the following cognitive domains: Reasoning ability (subtest 3 of the Leistungsprüfsystem, a German test covering Thurstone's primary mental abilities), executive functioning (difference of execution times between Trail Making Test A and B), working memory (Letter Number Sequencing), processing speed (Digit Symbol Coding), verbal memory (Verbal Learning and Memory Test) and divided attention (test battery for attention). 'The fine motor function composite consists of dotting and tapping subtests of the MacQuarrie test for mechanical ability. ${ }^{\mathrm{d} C o n t r o l}$ variables. 
Table 4 Severely impaired interpretation performers (LIP) show compromised cognition compared to healthy controls (Con) and non impaired interpretation performers (HIP)

\begin{tabular}{|c|c|c|c|c|c|c|c|c|}
\hline \multirow{2}{*}{$\frac{\text { Trait }}{\text { Basic characteristics }}$} & \multicolumn{3}{|c|}{ Descriptive } & \multirow[t]{2}{*}{ Group contrast } & \multirow[t]{2}{*}{ Effect } & \multirow[t]{2}{*}{ [95\%Cl] } & \multirow[t]{2}{*}{ Statistic } & \multirow[t]{2}{*}{$p$} \\
\hline & & & & & & & & \\
\hline Age (years) & $\mathrm{SZ}$ & $39.5 \pm 13$ & $(17-78)$ & & & & & \\
\hline \multirow[t]{3}{*}{ mean \pm SD (range) } & Con & $38.8 \pm 14$ & $(18-71)$ & HIP / Con & 2.75 & {$[-0.81,6.31]$} & $\mathrm{t}_{275}=1.522^{\mathrm{a}}$ & 0.13 \\
\hline & HIP & $41.0 \pm 11$ & $(22-64)$ & LIP / Con & 1.25 & {$[-2.31,4.81]$} & $\mathrm{t}_{275}=0.693^{\mathrm{a}}$ & 0.49 \\
\hline & LIP & $39.7 \pm 13$ & $(18-71)$ & LIP / HIP & -1.40 & {$[-4.58,1.77]$} & $\mathrm{t}_{174}=-0.873^{\mathrm{a}}$ & 0.38 \\
\hline \multirow[t]{5}{*}{ Gender (\% female) } & SZ & 33 & & & & & & \\
\hline & Con & 32 & & HIP / Con & OR 1.25 & {$[0.66,2.38]$} & Fisher $^{\mathrm{b}}$ & 0.54 \\
\hline & HIP & 38 & & LIP / Con & OR 0.83 & {$[0.42,1.62]$} & Fisher $^{b}$ & 0.64 \\
\hline & LIP & 28 & & LIP / HIP & OR 0.66 & {$[0.33,1.31]$} & Fisher $^{\mathrm{b}}$ & 0.26 \\
\hline & & & & & for females & & & \\
\hline \multirow[t]{5}{*}{ Smoker status (\% yes) } & $\mathrm{SZ}$ & 48 & & & & & & \\
\hline & Con & 63 & & HIP / Con & OR 0.45 & {$[0.21,0.94]$} & Fisher $^{b}$ & 0.025 \\
\hline & HIP & 43 & & LIP / Con & OR 0.57 & {$[0.26,1.23]$} & Fisher $^{\mathrm{b}}$ & 0.15 \\
\hline & LIP & 49 & & LIP / HIP & OR 1.26 & {$[0.52,3.05]$} & Fisher $^{\mathrm{b}}$ & 0.68 \\
\hline & & & & & for smokers & & & \\
\hline \multicolumn{9}{|c|}{ Disease related higher and basal cognition and premorbid intelligence } \\
\hline Reasoning $^{c}$ & SZ & $20.8 \pm 6.6$ & $(2-38)$ & & & & & \\
\hline \multirow[t]{3}{*}{ mean \pm SD (range) } & Con & $27.2 \pm 5.2$ & $(14-38)$ & HIP / Con & -3.00 & {$[-4.86,-1.14]$} & $\mathrm{t}_{265}=-3.180^{\mathrm{a}}$ & $1.6 \times 10^{-3}$ \\
\hline & HIP & $21.5 \pm 6.5$ & $(8-38)$ & LIP / Con & -5.49 & {$[-7.63,-3.35]$} & $\mathrm{t}_{265}=-5.058^{\mathrm{a}}$ & $7.9 \times 10^{-7}$ \\
\hline & LIP & $18.2 \pm 6.7$ & $(2-34)$ & LIP / HIP & -2.45 & {$[-4.07,-0.82]$} & $\mathrm{t}_{164}=-2.971^{\mathrm{a}}$ & $3.4 \times 10^{-3}$ \\
\hline Fine motor function & $\mathrm{SZ}$ & $-0.12 \pm 0.91$ & $(-3.22-3.22)$ & & & & & \\
\hline composite $^{c}$ & Con & $0.97 \pm 0.68$ & $(-1.09-2.83)$ & HIP / Con & -0.53 & {$[-0.75,-0.31]$} & $t_{268}=-4.674^{a}$ & $4.7 \times 10^{-6}$ \\
\hline \multirow[t]{2}{*}{ mean \pm SD (range) } & HIP & $-0.002 \pm 0.87$ & $(-2.40-1.83)$ & LIP / Con & -0.78 & {$[-1.03,-0.52]$} & $\mathrm{t}_{268}=-5.951^{\mathrm{a}}$ & $8.3 \times 10^{-9}$ \\
\hline & LIP & $-0.45 \pm 0.93$ & $(-2.89-1.90)$ & LIP / HIP & -0.23 & {$[-0.41,-0.05]$} & $\mathrm{t}_{167}=-2.471^{\mathrm{a}}$ & 0.014 \\
\hline Premorbid & $\mathrm{SZ}$ & $26.2 \pm 6.1$ & $(4-37)$ & & & & & \\
\hline intelligence $^{d}$ & Con & $30.9 \pm 4.0$ & $(18-37)$ & HIP / Con & -0.22 & {$[-2.06,1.62]$} & $t_{267}=-0.236^{a}$ & 0.81 \\
\hline \multirow[t]{2}{*}{ mean \pm SD (range) } & HIP & $27.8 \pm 5.0$ & $(13-37)$ & LIP / Con & -2.60 & {$[-4.74,-0.46]$} & $t_{267}=-2.393^{a}$ & 0.017 \\
\hline & LIP & $23.8 \pm 7.0$ & $(5-35)$ & LIP / HIP & -2.51 & {$[-4.19,-0.83]$} & $\mathrm{t}_{166}=-2.955^{\mathrm{a}}$ & $3.6 \times 10^{-3}$ \\
\hline
\end{tabular}

Multiple testing adjusted significances (applying Bonferroni ( $\mathrm{p} \leq 0.007)$ and the closed testing principle) are set in boldface. All other $\mathrm{p}$-values 0.05 are set in italic; ${ }^{a} t$-Statistic $t_{d f}$ with df degrees of freedom for estimation of difference in means (employing a linear model on Blom-transformed trait); ${ }^{b}$ Fisher's exact test for count data; ' Adjusted for age at exam and PANSS negative (value 7 for healthy control). Reasoning ability was measured by LPS3 (subtest 3 of the Leistungsprüfsystem, a German test covering Thurstone's primary mental abilities). Fine motor function composite is the mean from standardized (by Blom-transformation) MacQuarrie dotting and tapping; ${ }^{\mathrm{d}}$ Adjusted for age (age of onset of psychosis for schizophrenia HIP and LIP, age at exam for healthy control) and PANSS negative (value 7 for healthy control). Premorbid intelligence was assessed by MWTB (Mehrfachwahl-Wortschatz-Intelligenztest, English translation: multiple choice vocabulary test of intelligence). Abbreviations: $\mathrm{Con}=$ Healthy control $(\mathrm{N}=102)$; $\mathrm{SZ}=$ Descriptive statistics for whole schizophrenia sample $(\mathrm{N}=881)$; $\mathrm{HIP}=\mathrm{High}$ interpretation performers ( $\mathrm{N}=88)$; LIP= Low interpretation performers $(\mathrm{N}=88)$, OR=Odds Ratio.

in odor naming (average 0.42 points, $\mathrm{p}=0.043, \mathrm{t}_{978}=2.022$ ) but not interpretation.

\section{Odor naming and interpretation are impaired in schizophrenia patients}

The main study results are summarized in Figure $1 \mathrm{C}$. Inferior performance of schizophrenia patients $(\mathrm{N}=881)$ compared to 102 matched healthy controls became evident for odor interpretation and naming tasks (Figure 2A,B; interpretation: $\mathrm{p}=4.0 \times 10^{-10}$; naming: $\left.\mathrm{p}=2.9 \times 10^{-9}\right)$.
Odor naming and interpretation are differentially associated with psychopathology and cognition in schizophrenia patients

Odor naming and interpretation correlate substantially (Spearman correlation $\mathrm{r}=0.51$, 95\% CI [0.46, 0.56], $\left.\mathrm{p}<2.2 \times 10^{-16}\right)$. To investigate relative association strengths of odor naming and interpretation with 3 schizophrenia relevant symptom domains (cognition, negative and positive symptoms) and 2 control variables (fine motor function and premorbid intelligence) (Table 3), multiple linear regression was applied in the schizophrenia sample 
Table 5 Severely impaired interpretation performers (LIP) show a more severe psychopathology, compromised cognition and emotional processing compared to high interpretation performers (HIP)

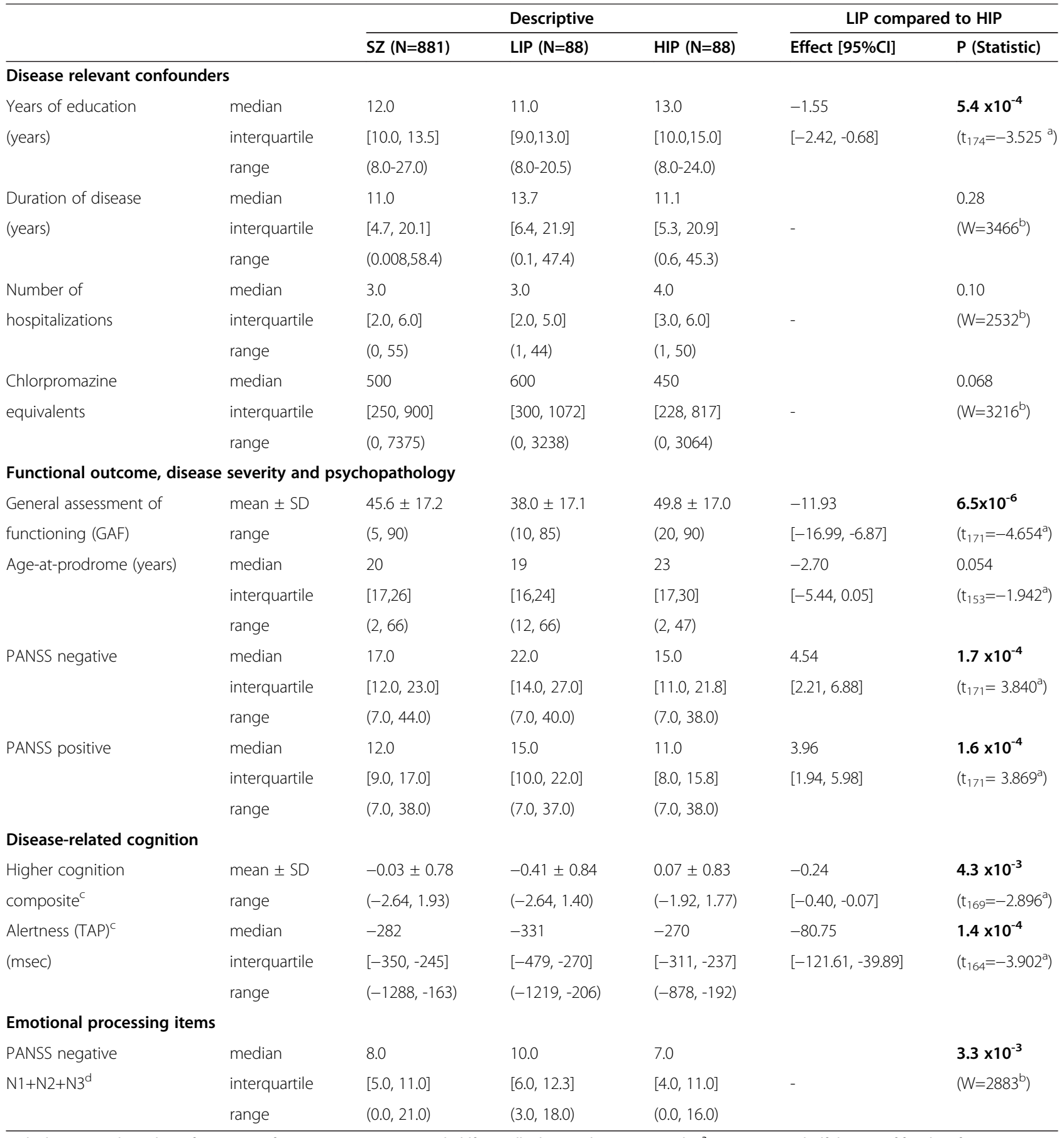

Multiple testing adjusted significances (Bonferroni: $\mathrm{p} \leq 0.0045$ ) are set in boldfaces, all other $\mathrm{p}$-values $\leq 0.05$ in italic. ${ }^{a}$ t-Statistic $t_{d f}$ with df degrees of freedom for estimation of difference in means (employing a linear model on Blom-transformed trait; small differences in degrees of freedom are due to low percentages of missing trait values).

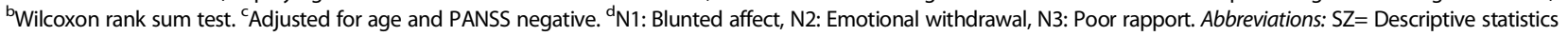
for whole schizophrenia sample $(\mathrm{N}=881)$; $\mathrm{HIP}=$ High interpretation performers $(\mathrm{N}=88)$; $\mathrm{LIP}=\mathrm{L}$ Low interpretation performers $(\mathrm{N}=88)$, $\mathrm{OR}=\mathrm{Odds}$ Ratio.

$(\mathrm{N}=881)$. Regression coefficients express relative association strength (relative to the standard deviation of the target trait). Odor naming and interpretation scores were strongly dependent on alertness. Worse naming results were additionally linked to impaired higher cognition and more severe negative symptoms (PANSS). Deficits in odor interpretation were associated with higher positive symptom severity (PANSS). No significant link was detected between odor naming or interpretation and fine motor function or premorbid intelligence. 


\section{Extreme group comparisons based on odor interpretation performance reveal strong differences in several lead symptom domains}

The frequency distributions of interpretation and naming scores (Figure 2A,B) showed increased probabilities of low scores for schizophrenia patients, especially for odor interpretation. This suggests that olfactory processing deficits may be particularly pronounced in a subgroup of schizophrenia patients. To achieve a maximum phenotypic contrast, schizophrenia high interpretation performers (HIP, scoring $>90^{\text {th }}$ percentile) were contrasted with low interpretation performers (LIP, scoring $<10^{\text {th }}$ percentile; $\mathrm{N}=88$ each) and compared to healthy controls (Con, $\mathrm{N}=102$ ) with respect to basic sample characteristics, disease related higher and basal cognition and premorbid intelligence. The 3 groups did not differ (pairwise comparisons) with regard to age, gender and smoking status. Expectedly, a highly significant naming deficit became obvious for LIP in comparison to HIP $\left(\mathrm{p}=7.0 \times 10^{-28}\right)$ and healthy controls $\left(\mathrm{p}=9.1 \times 10^{-29}\right)$ (Figure $2 \mathrm{C}$ ). In terms of reasoning capabilities, healthy controls obtained better results than schizophrenia HIP $\left(p=1.6 \times 10^{-3}\right)$ with the latter performing superior to LIP (Table 4, $\mathrm{p}=3.4 \times 10^{-3}$ ) (Figure 1C). For fine motor function, both schizophrenia groups performed comparably and markedly worse than healthy controls (HIP/LIP: $\mathrm{p}=0.015$; HIP/Con: $\mathrm{p}=4.4 \times 10^{-6}$; LIP/Con: $\left.\mathrm{p}=8.1 \times 10^{-9}\right)$. Also for premorbid intelligence, a difference was observed between disease groups (HIP/ Con: $p=0.81$; LIP/Con: $p=0.017$; LIP/HIP: $p=3.6 \times 10^{-3}$ ) (Table 4).

More detailed assessment of group contrast between schizophrenia LIP compared to HIP in disease-related domains, revealed higher severity of negative symptoms $\left(\mathrm{p}=1.7 \times 10^{-4}\right)$ and positive symptoms $\left(\mathrm{p}=1.6 \times 10^{-4}\right)$ for LIP (Figure $1 \mathrm{C}$ ). Additionally, strongly impaired cognitive and emotional processing became evident (higher cognition composite: $\mathrm{p}=3.7 \times 10^{-3}$; alertness: $\mathrm{p}=1.4 \times 10^{-4}$; emotional processing: $\mathrm{p}=3.3 \times 10^{-3}$ ), resulting in a worse functional outcome ( $\mathrm{p}=6.5 \times 10^{-6}$; average difference 12 GAF units) for LIP (Table 5). Extreme group comparison for individual neuropsychological measures (Table 2) revealed strongest differences for reasoning $\left(\mathrm{p}=3.6 \times 10^{-3}\right)$ followed by processing speed $(\mathrm{p}=0.011)$ and working memory $(\mathrm{p}=0.049)$. Results for executive functioning, verbal memory and divided attention failed to be significant. No significant differences were observed with regard to possible confounders indicating overall disease severity (duration of disease, number of hospitalizations and chlorpromazine equivalents). Also, age-at-prodrome (median, range for LIP: 19, 12-66, for HIP: 23, 2-47, $\mathrm{p}=0.054$ ) and age-of-onset (LIP: 23, 15-67, HIP: 27, 8-55, p=0.0079) were only close to or nominally significant, i.e. above the Bonferroni corrected significance threshold ( $p>0.005)$. Together, the results of the extreme group approach indicate that deficits in odor interpretation are accompanied by more severe psychopathology, worse cognitive and emotional processing and inferior functional outcome.

\section{Discussion}

The present study, building on and extending previous work, demonstrates in a large sample of subjects that the ability to name and qualitatively interpret odors is markedly impaired in schizophrenia. Importantly, the study adds new aspects to the available literature by showing distinct associations between central olfactory measures and schizophrenia-relevant clinical variables. Extreme group comparisons providing maximum phenotypic contrast deliver further evidence for a biological subgroup of patients, defined by a severe olfactory dysfunction. This subgroup is characterized by profound abnormalities in higher olfactory processing, together with a more severe psychopathology, remarkable cognitive deficits across various neuropsychological measures and pronounced affective flattening, resulting in an overall inferior functional outcome as compared to all other schizophrenia patients.

\section{Odor naming ability relies on higher cognitive processing}

Odor naming but not interpretation was found to depend on the integrity of higher cognitive processing when analyzed in all 881 patients. In contrast to Saoud and coworkers, who investigated only a small number of individuals [16], we found odor naming, as almost all neuropsychological tests [26], to correlate with PANSS negative scores. Due to the low number of odors used for the naming task in the present work, distributions of odor naming sum scores were not considered suitable for the definition of extreme groups. Instead, the definition was based on interpretation performance. Future studies will have to aim at doubling the number of odors to be able to thoroughly explore construct validity of the odor naming task.

\section{Positive symptoms interfere with odor interpretation ability}

By applying multiple linear regression analyses to investigate relative association strengths of olfactory target measures with several symptom domains, odor interpretation, but not naming, was found to be influenced by the severity of positive symptoms. In fact, while the relationship between basic olfactory processing (e.g. passive odor identification) and severe negative symptoms is quite well established [37-41], this is the first study to provide evidence for psychotic symptoms to interfere with performance in an olfactory function test. This relationship is supported by imaging studies showing that functional abnormalities in temporo-limbic regions are related to altered familiarity and hedonicity judgements in schizophrenia 
[42]. Disconnectivity in these brain regions also contributes to the emergence of hallucinations in schizophrenia $[43,44]$. Based on these findings future studies should address whether the relationship of odor interpretation and positive symptom severity is mediated by a dysfunctional integration of sensory input into cognitive-emotional processing. The potential for odor interpretation tasks to indirectly assess the predisposition to develop positive symptoms should also be further evaluated.

\section{Alertness modulates performance in central olfactory measures}

The large amount of variance shared by odor naming and interpretation can be partially explained by the strong association of both olfactory tasks with the level of alertness. The latter represents the intensity aspect of attention and comprises both a state of general wakefulness (intrinsic alertness) and the ability to increase the readiness to react in response to a cue over a short period of time (phasic alertness) [45]. Importantly, attention is a requirement for assigning the continuous flow of air through the nose to either respiration or olfactory exploration [46]. Non-attended, the olfactory content of inhalations is ignored and not processed further. Effects of attentional modulation on activity in human primary [46] and secondary olfactory cortices [47] and amygdala [48] support this notion. Consequently, the extent to which an individual attends to a presented odor and is able to maintain the level of alertness during the completion of a task, likely influences the amount of resources provided for higherorder mental operations following the perception of an olfactory stimulus. Additionally, it is well established that olfactory (spicy, fruity) and gustatory (salty, sweet) judgements lead to the activation of associated semantic networks. This results in a facilitation of olfactory naming - at least in healthy individuals [25]. However, as the here applied odor interpretation task was the same for all odors, differential depth-of-processing effects, potentially confounding odor naming results, can be excluded.

\section{Odor interpretation is associated with discrete neuropsychological functions}

Comparisons of low and high odor interpretation performers (HIP / LIP) with regard to single neuropsychological measures revealed largest group differences for reasoning abilities. Processing speed and working memory also differed significantly dependent on odor interpretation performance whereas executive functioning (set shifting), verbal memory and divided attention did not. Together, these findings should stimulate further research into primary cognitive functions involved in the odor interpretation task.

Of note is the fact that premorbid intelligence, ageat-prodrome and age-of-onset of schizophrenia showed at least a strong tendency of a difference between LIP and HIP, indicating a potentially more pronounced neurodevelopmental aspect of the disease in LIP. This difference alone, however, is unlikely to explain the marked contrast in psychopathology and cognition between extreme groups since post hoc linear regression models remained significant upon inclusion of neurodevelopmental variables as covariates (data not shown).

\section{Conclusions}

Although further work is needed to improve psychometric properties of the measures by e.g. increasing the number of applied odors, we deliver first evidence for both tasks to accentuate slightly dissimilar aspects of higher brain functioning. Future studies are needed to delineate whether prevailing impairment in either odor naming or interpretation indicates a preferential lack of integrity in either sensory-cognitive or sensory-emotional brain networks. If developed into psychometrically sound tests, odor naming and interpretation tasks could assist the delineation of biologically defined disease subphenotypes, characterized by preponderance of either cognitive decline/ negative symptoms, or of pronounced positive symptoms.

\section{Competing interest \\ The authors declare that they have no competing interests.}

\section{Authors' contributions}

AK, MB and HE developed the olfactory tasks. Phenotype data were collected and entered into the database by $\mathrm{CH}, \mathrm{MB}$ and $\mathrm{AK}$. DM and AK performed all statistical analyses and designed manuscript and figures. HE guided the project and data analysis, together with $\mathrm{HB}$. AK, together with $\mathrm{HE}$, wrote the paper. All authors discussed the results, read and commented on the manuscript, and have seen and approved the final version.

\section{Acknowledgements}

We are indebted to all healthy individuals and all patients for their participation in the study, and all collaborating GRAS centers for their support. We are grateful to all colleagues who contributed to the GRAS data collection.

\section{Funding}

This work was supported by the Max Planck Society, the Max-PlanckFörderstiftung, and by the DFG Center for Nanoscale Microscopy \& Molecular Physiology of the Brain (CNMPB)

\section{Author details}

'Clinical Neuroscience, Max Planck Institute of Experimental Medicine, Hermann-Rein-Str.3, 37075 Göttingen, GERMANY. ²Department of Genetic Epidemiology of the University Medical Center, Göttingen, Germany. ${ }^{3}$ DFG Research Center for Nanoscale Microscopy \& Molecular Physiology of the Brain (CNMPB), Göttingen, Germany.

Received: 26 June 2013 Accepted: 20 August 2013

Published: 30 August 2013

\section{References}

1. Kivity S, Ortega-Hernandez OD, Shoenfeld Y: Olfaction-a window to the mind. Isr Med Assoc J 2009, 11(4):238-243.

2. Benarroch EE: Olfactory system: functional organization and involvement in neurodegenerative disease. Neurology 2010, 75(12):1104-1109.

3. Turetsky BI, Hahn CG, Borgmann-Winter K, Moberg PJ: Scents and nonsense: olfactory dysfunction in schizophrenia. Schizophr Bull 2009, 35(6):1117-1131. 
4. Larsson M, Willander J: Autobiographical odor memory. Ann N Y Acad Sci 2009, 1170:318-323.

5. Willander J, Larsson M: Olfaction and emotion: the case of autobiographical memory. Mem Cognit 2007, 35(7):1659-1663.

6. Savic I: Processing of odorous signals in humans. Brain Res Bull 2001, 54(3):307-312

7. Atanasova B, Graux J, El Hage W, Hommet C, Camus V, Belzung C: Olfaction: a potential cognitive marker of psychiatric disorders. NeurosC Biobehav Rev 2008, 32(7):1315-1325.

8. Moberg PJ, Arnold SE, Doty RL, Gur RE, Balderston CC, Roalf DR, Gur RC, Kohler CG, Kanes SJ, Siegel SJ, et al: Olfactory functioning in schizophrenia: relationship to clinical, neuropsychological, and volumetric MRI measures. J Clin Exp Neuropsychol 2006, 28(8):1444-1461.

9. Arnold SE, Han LY, Moberg PJ, Turetsky BI, Gur RE, Trojanowski JQ, Hahn CG: Dysregulation of olfactory receptor neuron lineage in schizophrenia. Arch Gen Psychiatry 2001, 58(9):829-835.

10. Turetsky BI, Hahn CG, Arnold SE, Moberg PJ: Olfactory receptor neuron dysfunction in schizophrenia. Neuropsychopharmacology 2009, 34(3):767-774

11. Doty RL, Shaman P, Kimmelman CP, Dann MS: University of Pennsylvania Smell Identification Test: a rapid quantitative olfactory function test for the clinic. Laryngoscope 1984, 94(2 Pt 1):176-178.

12. Moberg PJ, Doty RL, Turetsky BI, Arnold SE, Mahr RN, Gur RC, Bilker W, Gur RE: Olfactory identification deficits in schizophrenia: correlation with duration of illness. Am J Psychiatry 1997, 154(7):1016-1018.

13. Striebel KM, Beyerstein B, Remick RA, Kopala L, Honer WG: Olfactory identification and psychosis. Biol Psychiatry 1999, 45(11):1419-1425.

14. Doty RL, Kerr KL: Episodic odor memory: influences of handedness, sex, and side of nose. Neuropsychologia 2005, 43(12):1749-1753.

15. Schab FR: Odors and the remembrance of things past. J Exp Psychol Learn Mem Cogn 1990, 16(4):648-655

16. Saoud M, Hueber T, Mandran H, Dalery J, D'Amato T: Olfactory identification deficiency and WCST performance in men with schizophrenia. Psychiatry research 1998, 81(2):251-257.

17. Good KP, Tibbo P, Milliken H, Whitehorn D, Alexiadis M, Robertson N, Kopala $L C$ : An investigation of a possible relationship between olfactory identification deficits at first episode and four-year outcomes in patients with psychosis. Schizophr Res 2010, 124(1-3):60-65.

18. Ishizuka K, Tajinda K, Colantuoni C, Morita M, Winicki J, Le C, Lin S, Schretlen D, Sawa A, Cascella NG: Negative symptoms of schizophrenia correlate with impairment on the University of Pennsylvania smell identification test. Neurosci Res 2010, 66(1):106-110.

19. Seidman L, Talbot NL, Kalinowski AG, McCarley RW, Faraone SV, Kremen WS, Pepple JR, Tsuang MT: Neuropsychological probes of fronto-limbic system dysfunction in schizophrenia. Olfactory identification and Wisconsin Card Sorting performance. Schizophr Res 1991, 6(1):55-65.

20. Soudry Y, Lemogne C, Malinvaud D, Consoli SM, Bonfils P: Olfactory system and emotion: common substrates. Eur Ann Otorhinolaryngol Head Neck Dis 2011, 128(1):18-23.

21. Hudry J, Saoud M, D'Amato T, Dalery J, Royet JP: Ratings of different olfactory judgements in schizophrenia. Chem Senses 2002, 27(5):407-416.

22. Kamath V, Moberg PJ, Kohler CG, Gur RE, Turetsky BI: Odor Hedonic Capacity and Anhedonia in Schizophrenia and Unaffected First-Degree Relatives of Schizophrenia Patients. Schizophr Bull 2011, 187(1-2):30-35.

23. Kamath $\mathrm{V}$, Turetsky BI, Moberg PJ: Identification of pleasant, neutral, and unpleasant odors in schizophrenia. Psychiatry research 2011, 187(1-2):30-35.

24. Moberg PJ, Arnold SE, Doty RL, Kohler C, Kanes S, Seigel S, Gur RE, Turetsky BI: Impairment of odor hedonics in men with schizophrenia. Am J Psychiatry 2003, 160(10):1784-1789.

25. Royet JP, Koenig O, Paugam-Moisy H, Puzenat D, Chasse JL: Levels-ofprocessing effects on a task of olfactory naming. Percept Mot Skills 2004, 98(1):197-213

26. Ribbe K, Friedrichs H, Begemann M, Grube S, Papiol S, Kastner A, Gerchen MF, Ackermann V, Tarami A, Treitz A, et al: The cross-sectional GRAS sample: a comprehensive phenotypical data collection of schizophrenic patients. BMC Psychiatry 2010, 10:91

27. Doty RL, Shaman P, Dann M: Development of the University of Pennsylvania Smell Identification Test: a standardized microencapsulated test of olfactory function. Physiol Behav 1984, 32(3):489-502
28. Wright HN: Characterization of olfactory dysfunction. Arch Otolaryngol Head Neck Surg 1987, 113(2):163-168.

29. Symonds PM: Factors influencing test reliability. J Educ Psychol 1928, 14(2):73-87. XIX

30. Kay SR, Fiszbein A, Opler LA: The positive and negative syndrome scale (PANSS) for schizophrenia. Schizophr Bull 1987, 13(2):261-276.

31. Zimmermann P, Fimm B: Testbatterie zur'Aufmerksamkeitsprüfung (TAP). Version 1.02C. PSYTEST: Herzogenrath; 1993.

32. Chapman RL: The MacQuarrie test for mechanical ability. Psychometrika 1948, 13(3):175-179

33. Lehrl S, Triebig G, Fischer B: Multiple choice vocabulary test MWT as a valid and short test to estimate premorbid intelligence. Acta neurologica Scandinavica 1995, 91(5):335-345.

34. Rauch AV, Reker M, Ohrmann P, Pedersen A, Bauer J, Dannlowski U, Harding L, Koelkebeck K, Konrad C, Kugel H, et al: Increased amygdala activation during automatic processing of facial emotion in schizophrenia. Psychiatry research 2010, 182(3):200-206

35. Horn W: Leistungsprüfsystem (LPS). 2nd edition. Goettingen; 1983.

36. Blom G: Statistical estimates and transformed beta-variables. New York: Wiley; 1958.

37. Brewer WJ, Edwards J, Anderson V, Robinson T, Pantelis C Neuropsychological, olfactory, and hygiene deficits in men with negative symptom schizophrenia. Biol Psychiatry 1996, 40(10):1021-1031.

38. Brewer WJ, Wood SJ, Pantelis C, Berger GE, Copolov DL, McGorry PD: Olfactory sensitivity through the course of psychosis: Relationships to olfactory identification, symptomatology and the schizophrenia odour. Psychiatry research 2007, 149(1-3):97-104.

39. Corcoran C, Whitaker A, Coleman E, Fried J, Feldman J, Goudsmit N, Malaspina D: Olfactory deficits, cognition and negative symptoms in early onset psychosis. Schizophr Res 2005, 80(2-3):283-293.

40. Malaspina D, Coleman E: Olfaction and social drive in schizophrenia. Arch Gen Psychiatry 2003, 60(6):578-584.

41. Malaspina D, Coleman E, Goetz RR, Harkavy-Friedman J, Corcoran C, Amador $X$, Yale S, Gorman JM: Odor identification, eye tracking and deficit syndrome schizophrenia. Biol Psychiatry 2002, 51(10):809-815.

42. Saoud M, Royet JP, Plailly J, D'Amato T: Left temporo-limbic and orbital dysfunction in schizophrenia during odor familiarity and hedonicity judgments. Neuroimage 2006, 29(1):302-313.

43. Ffytche DH: The hodology of hallucinations. Cortex 2008, 44(8):1067-1083.

44. Lawrie SM, Buechel C, Whalley HC, Frith CD, Friston KJ, Johnstone EC: Reduced frontotemporal functional connectivity in schizophrenia associated with auditory hallucinations. Biol Psychiatry 2002, 51(12):1008-1011.

45. Sturm W, de Simone A, Krause BJ, Specht K, Hesselmann V, Radermacher I, Herzog H, Tellmann L, Muller-Gartner HW, Willmes K: Functional anatomy of intrinsic alertness: evidence for a fronto-parietal-thalamic-brainstem network in the right hemisphere. Neuropsychologia 1999, 37(7):797-805.

46. Zelano C, Bensafi M, Porter J, Mainland J, Johnson B, Bremner E, Telles C, Khan $\mathrm{R}$, Sobel N: Attentional modulation in human primary olfactory cortex. Nat Neurosci 2005, 8(1):114-120.

47. O'Doherty J, Rolls ET, Francis S, Bowtell R, McGlone F, Kobal G, Renner B, Ahne G: Sensory-specific satiety-related olfactory activation of the human orbitofrontal cortex. Neuroreport 2000, 11(4):893-897.

48. Gottfried JA, O'Doherty J, Dolan RJ: Encoding predictive reward value in human amygdala and orbitofrontal cortex. Science 2003, 301(5636):1104-1107.

\section{doi:10.1186/1471-244X-13-218}

Cite this article as: Kästner et al: Odor naming and interpretation performance in 881 schizophrenia subjects: association with clinical parameters. BMC Psychiatry 2013 13:218. 